\title{
Characterization of Pareto Distribution Through Expectation of Function of Order Statistics
}

\author{
Bhatt Milind. B \\ Department of Statistics \\ Sardar Patel University \\ Vallabhvidyanagar-388129 \\ Dist. Anand, Gujarat \\ INDIA \\ bhattmilind_b@yahoo.com
}

\begin{abstract}
For characterization of Pareto distribution one needs any arbitrary non constant function only by approach of identity of distribution and equality of expectation of function of random variable in place of approaches such as relation (linear) in (economic variation) reported and true income, independency of suitable function of order statistics, mean and the extreme observation of the sample etc. Examples are given for illustrative purpose.
\end{abstract}

Keyword: Characterization; Pareto distribution

\section{Introduction}

Certain skew pattern appear in socioeconomic quantities such stock price fluctuation, personal income, economic variation in reported income and under-reporting error [See. Krishnaji (1970), Nagesh (1974)] have certain invariant properties for which Pareto distribution foun most duitable. Amongst many other Pareto distribution used to study skew pattern. Pareto distribution also used to study empiric phenomena such as occurrence of natural resources, error clustering in communication circuit, size of firm, city, population and reliability theory.

Independence of suitable function of order statistics was used for characterization of Pareto distribution by Henrick (1970), Ahsanullah (1973, 1974 ), Shah (1981) and Dimaki (1993) where as Srivastava (1976) used mean and the extreme observation of the sample.

Other attempts were made for characterization of exponential and related distributions assuming linear relation of conditional expectation by Beg (1974) and Dallas (1976), characterization of some types of distributions using recurrence relations between expectations of function of order statistics by Alli (1998) and characterization results on exponential and related distributions by Tavangar (2010) included characterization of Pareto distribution.

This research note provides the characterization based on identity of distribution and equality of expectation of function of order statistics for Pareto distribution with the probability density function (p.d.f.).

Corresponding author: Bhatt Milind. B, Department of Statistics, Sardar Patel University, Vallabhvidyanagar-388129, Dist. Anand, Gujarat, INDIA. E-mail: bhattmilind_b@yahoo.com. 
This work was supported by UGC Major Research Project : F.No.42-39/2013(SR) dated 12-3-2013

This research note provides characterization of Pareto distribution with probability density function (pdf)

$$
f(x, \theta)=\left\{\begin{array}{c}
\frac{c \theta^{c}}{x^{c+1}} ; \theta<x<\infty ; c>0, \\
0 ; \text { otherwise, }
\end{array}\right.
$$

where $-\infty \leq a<b \leq \infty$ are known constants and $(1 / x)^{c+1}$ is positive absolutely continuous function and $c \theta^{c}$ is everywhere differentiable function is characterized.

Note that $c$ is income concentration use as measure of inequalities in income distribution and $\theta$ is minimum level of income.

The aim of the present research note is to give path braking new characterization for Pareto distribution defined in (1.1) through expectation of function of order statistics, using identity and equality of expectation. Characterization theorem provrd in section 2 with method for characterization as remark and section 3 devoted to applications for illustrative purpose.

\section{Characterization Theorem}

Let $X_{1}, X_{2}, \ldots, X_{n}$ be a random sample of size $n$ from distribution function $f$ and let $X_{1: n}<X_{2: n}<$ $\ldots<X_{n n \text { : }}$ be the set of corresponding order statistics. Assume that $f$ is continuous on the interval $(a, b)$ where $-\infty \leq a<b \leq \infty$. Let $g\left(X_{1: n}\right)$ and $\phi\left(X_{1: n}\right)$ be be two distinct differentiable and intregrable functions of first order statistic; $X_{1: n}$ on the interval $(a, b)$ where $-\infty \leq a<b \leq \infty$. and moreover $\$ g\left(X_{1: n}\right)$ be non-constant function of $X_{1: n}$. Then

$$
E\left[g\left(X_{1: n}\right)-\left(\frac{X_{1: n}}{\mathrm{nc}}\right) \frac{d}{d X_{1: n}} g\left(X_{1: n}\right)\right]=g(\theta) \ldots
$$

is the necessary and sufficient condition for pdf $f$ of $F$ to be $f(x, \theta)$ defined in (1.1).

Proof. Given $f(x, \theta)$ defined in (1.1), for necessity of (2.1) if $\phi\left(X_{1: n}\right)$ is such that $g(\theta)=E \phi\left(X_{1: n}\right)$ where $g(\theta)$ is differentiable function then using $f\left(X_{1: n} ; \theta\right)$ pdf of first order statistic; $X_{1: n}$ one gets

$$
g(\theta)=\int_{\theta}^{b} \phi\left(x_{1: n}\right) f\left(x_{n: n}, \theta\right) d x_{n: n} \cdots
$$

Differentiating (2.2) with respect to $\theta$ on both sides and replacing $X_{1: n}$ for $\theta$ and simplifying one gets

$$
\phi\left(\mathrm{x}_{1: \mathrm{n}}\right)=g\left(X_{1: n}\right)-\left(\frac{X_{1: n}}{\mathrm{nc}}\right) \frac{d}{d X_{1: n}} g\left(X_{1: n}\right) \ldots
$$

which establishes necessity of (2.1). Conversely given (2.1), let $k\left(x_{1: n} ; \theta\right)$ be the pdf of first order statistic; $X_{1: n}$ such that

$$
g(\theta)=\int_{\theta}^{b} \phi\left(x_{1: n}\right) k\left(x_{1: n}, \theta\right) d x_{1: n} \ldots
$$

Since $\left(n c x_{n: n}^{n c}\right) ;(n \geq 1)$ is increasing integrable and differentiable function on the interval $(a, b)$ with $\left(\frac{1}{n c b^{n c}}\right)=0$ the following identity holds

$$
g(\theta) \equiv-n c \theta^{n c} \int_{\theta}^{b}\left[\frac{d}{d x_{1: n}}\left\{g\left(x_{1: n}\right)\left(\frac{1}{n c x_{1: n}^{n c}}\right)\right\}\right] d x_{1: n} \ldots
$$

Differentiating integrand $g\left(x_{1: n}\right)\left(\frac{1}{n c x_{1: n}^{n c}}\right)$ with respect to $x_{1: n}$ and simplifying after taking $\frac{d}{d x_{1: n}}\left(\frac{1}{n c x_{1: n}^{n c}}\right)$ 
as one factor one gets $(2.5)$ as

$$
g(\theta) \equiv \int_{\theta}^{b}\left[g\left(X_{1: n}\right)+\frac{\left(\frac{X_{1: n}}{n c}\right)}{\frac{d}{d X_{1: n}}\left(\frac{1}{n c x_{1: n}^{n c}}\right)} \frac{d}{d X_{1: n}} g\left(X_{1: n}\right)\right]\left\{-n c \theta^{n c} \frac{d}{d X_{1: n}}\left(\frac{1}{n c x_{1: n}^{n c}}\right)\right\} d x_{1: n} \ldots
$$

and substituting derivative of $\left(\frac{1}{n c x_{1: n}^{n c}}\right)$ in (2.6) one gets

$$
g(\theta) \equiv \int_{\theta}^{b} \phi\left(\mathrm{x}_{1: \mathrm{n}}\right) \frac{n c \theta^{n c}}{x_{1: n}^{n c+1}} d x_{1: n} \cdots
$$

where $\phi\left(\mathrm{x}_{1: \mathrm{n}}\right)$ is as derived in (2.3). By uniqueness theorem from (2.4) and (2.7)

$$
\mathrm{k}\left(\mathrm{x}_{1: \mathrm{n}}, \theta\right)=\frac{n c \theta^{n c}}{x_{1: n}^{n c+1}} \ldots
$$

Since $\left(n c x_{1: n}^{n c}\right) ;(n \geq 1)$ is increasing integrable and differentiable function on the interval $(a, b)$ with $\left(\frac{1}{n c b^{n c}}\right)=0$ and since $\left(n c x_{1: n}^{n c}\right)$ is increasing function for $-\infty \leq a<b \leq \infty$ with $\left(\frac{1}{n c b^{n c}}\right)=0$. is satisfy only when range of $\mathrm{X}_{1: \mathrm{n}}$ is truncated by $\theta$ from left and integrating $(2.8)$ on the interval $(\theta, b)$ on both sides, one gets

$$
1=\int_{\theta}^{\mathrm{b}} \mathrm{k}\left(\mathrm{x}_{1: \mathrm{n}}, \theta\right) \mathrm{dx}_{1: \mathrm{n}}
$$

For $n=1,\left[k\left(\mathrm{x}_{1: \mathrm{n}}, \theta\right)\right]_{n=1}$ reduces to $f(x, \theta)$ defined in (1.1). Hence sufficiency of (2.1) is established.

Remark 2.1. Using $\phi(X)$ given in (2.2) one can determine $f(x, \theta)$ by

$$
M\left(\mathrm{X}_{1: \mathrm{n}}\right)=\frac{\frac{d}{d \mathrm{X}_{1: \mathrm{n}}} g\left(\mathrm{X}_{1: \mathrm{n}}\right)}{\phi\left(\mathrm{X}_{1: \mathrm{n}}\right)-g\left(\mathrm{X}_{1: \mathrm{n}}\right)} \cdots
$$

and pdf is given by

$$
\mathrm{f}(x, \theta)=\left[-\frac{\frac{\mathrm{d}}{\mathrm{dx}_{1: \mathrm{n}}} T\left(\mathrm{x}_{1: \mathrm{n}}\right)}{T(\theta)}\right]_{\mathrm{n}=1}, \mathrm{a}<\theta<x<b \quad \ldots
$$

where $T(X)$ is decreasing function for $-\infty \leq a<b \leq \infty$ with $T(b)=0$ such that it satisfies

$$
\mathrm{M}\left(X_{1: n}\right)=\frac{\mathrm{d}}{\mathrm{d} X_{1: n}}\left[\log T\left(X_{1: n}\right)\right] \ldots
$$

Remark 2.1. The theorem 2.1 for function of first order statistics with remark 2.1also holds for random variable $X$ when $(n=1)$.

3. Examples. Using method describe in remark 2.1 Pareto distribution through expectation of non-constant function of order statistics is characterized as illustrative example and significant of unified approach of characterization result.

Example 3.1 Characterization of Pareto distribution through the Minimum Variance Unbiased (UMVU) estimator $\widehat{e^{\theta}}$ of $e^{\theta}$ is given.

$$
\widehat{e^{\theta}}=e^{X_{1: n}}\left(1-\frac{X_{1: n}}{\mathrm{nc}}\right)=\mathrm{g}\left(X_{1: n}\right)
$$

Using (2.3) one gets 


$$
\phi\left(\mathrm{x}_{1: \mathrm{n}}\right)=g\left(X_{1: n}\right)-\left(\frac{X_{1: n}}{\mathrm{nc}}\right) \frac{d}{d X_{1: n}} g\left(X_{1: n}\right)=e^{X_{1: n}}\left(1-2 \frac{X_{1: n}}{\mathrm{nc}}+\left(\frac{X_{1: n}}{\mathrm{nc}}\right)^{2}+\frac{X_{1: n}}{(\mathrm{nc})^{2}}\right)
$$

and (2.9) of remark 2.1

$$
M\left(\mathrm{X}_{1: \mathrm{n}}\right)=\frac{\frac{d}{d \mathrm{X}_{1: \mathrm{n}}} g\left(\mathrm{X}_{1: \mathrm{n}}\right)}{\phi\left(\mathrm{X}_{1: \mathrm{n}}\right)-g\left(\mathrm{X}_{1: \mathrm{n}}\right)}=-\frac{\mathrm{nc}}{X_{1: n}}
$$

with

$$
\frac{d}{d x_{1: n}}\left[\log \left(\frac{1}{n c x_{1: n}^{n c}}\right)\right]=M\left(\mathrm{X}_{1: n}\right)
$$

then

$$
\mathrm{T}\left(x_{1: n}\right)=-\frac{1}{x_{1: n}^{n c+1}}
$$

and

$$
\mathrm{f}(x, \theta)=\left[-\frac{\frac{\mathrm{d}}{\mathrm{dx}: \mathrm{n}}\left(\mathrm{x}_{1: \mathrm{n}}\right)}{T(\theta)}\right]_{\mathrm{n}=1}=\frac{c \theta^{c}}{x^{c+1}}, \mathrm{a}<\theta<x<b
$$

Example 3.2 Characterization of Pareto distribution through the uniformly minimum variance unbiased (UMVU) estimator $\hat{g}(\theta)$ and maximum likelihood estimator (MLE) $\tilde{g}(\theta)$ of $g(\theta)$ such as $\mu_{1}^{\prime}(\theta)$; mean, $\mu_{1}^{\prime}(\theta) ; \mathrm{r}^{\text {th }}$ moment $e^{\theta}, e^{-\theta}, Q_{p}(\theta)$; $\mathrm{p}^{\text {th }}$ quantile, $F(t)$; distribution function and $\bar{F}(t)$; reliability function is given. For the (UMVU) estimator

$$
\hat{g}_{i}(\theta)=\left\{\begin{array}{c}
\hat{\mu}_{1}^{\prime}(\theta)=\frac{c}{\mathrm{c}-1}\left[1-\frac{1}{\mathrm{nc}}\right] X_{1: n} ; \text { for } i=1 \\
\hat{\mu}_{r}^{\prime}(\theta)=\frac{c}{\mathrm{c}-\mathrm{r}}\left[1-\frac{r}{\mathrm{nc}}\right] X_{1: n}^{r} ; \text { for } i=2 \\
\widehat{e^{\theta}}=e^{X_{1: n}}\left(1-\frac{X_{1: n}}{\mathrm{nc}}\right) ; \text { for } i=3 \\
\widehat{e^{-\theta}}=e^{-X_{1: n}}\left(1+\frac{X_{1: n}}{\mathrm{nc}}\right) ; \text { for } i=4 \\
\hat{Q}_{p}(\theta)=X_{1: n}(1-p)^{-\frac{1}{c}}\left\{1-\frac{1}{\mathrm{nc}}\right\} ; \text { for } i=5 \\
\hat{F}(t)=1-\left(\frac{X_{1: n}}{t}\right)^{c}\left\{1-\frac{1}{\mathrm{n}}\right\} ; \text { for } i=6 \\
\hat{\bar{F}}(t)=\left(\frac{X_{1: n}}{t}\right)^{c}\left\{1-\frac{1}{\mathrm{nc}}\right\} ; \text { for } i=7
\end{array}\right.
$$

and MLE 


$$
\tilde{g}_{i}(\theta)=\left\{\begin{array}{c}
\tilde{\mu}_{1}^{\prime}(\theta)=\frac{c}{c-1} X_{1: n} ; \text { for } i=1 \\
\tilde{\mu}_{r}^{\prime}(\theta)=\frac{c}{c-r} X_{1: n}^{r} ; \text { for } i=2 \\
\widetilde{e^{\theta}}=e^{X_{1: n}} ; \text { for } i=3 \\
\widetilde{e^{-\theta}}=e^{-X_{1: n}} ; \text { for } i=4 \\
\tilde{Q_{p}(\theta)}=X_{1: n}(1-p)^{-\frac{1}{c}} ; \text { for } i=5 \\
\tilde{F}(t)=1-\left(\frac{X_{1: n}}{t}\right)^{c} ; \text { for } i=6 \\
\tilde{\bar{F}}(t)=\left(\frac{X_{1: n}}{t}\right)^{c} ; \text { for } i=7
\end{array}\right.
$$

One gets

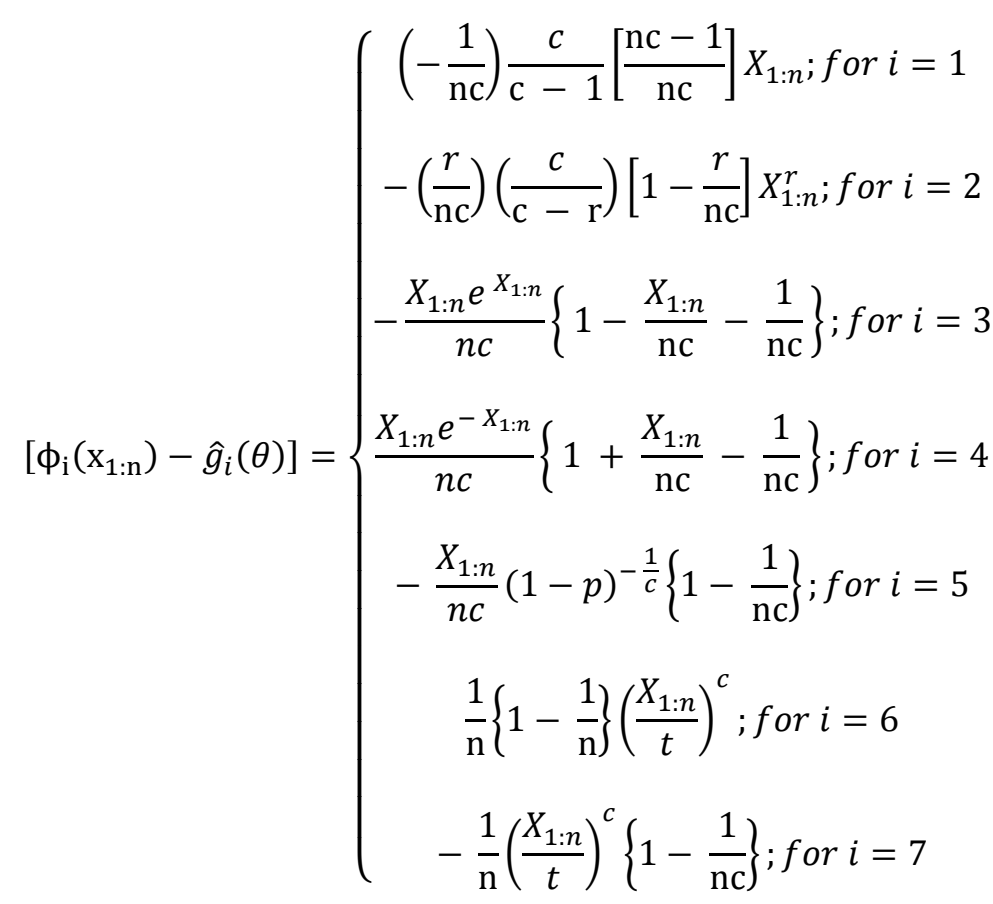

and 


$$
\left[\phi_{\mathrm{i}}\left(\mathrm{x}_{1: \mathrm{n}}\right)-\tilde{g}_{i}(\theta)\right]=\left\{\begin{array}{c}
-\frac{c}{\mathrm{c}-1}\left(\frac{X_{1: n}}{\mathrm{nc}}\right) ; \text { for } i=1 \\
-\left(\frac{c}{\mathrm{c}-\mathrm{r}}\right) \frac{r X_{1: n}^{r}}{\mathrm{nc}} ; \text { for } i=2 \\
-\frac{X_{1: n} e^{X_{1: n}}}{\mathrm{nc}} ; \text { for } i=3 \\
\frac{X_{1: n} e^{-X_{1: n}}}{\mathrm{nc}} ; \text { for } i=4 \\
-\frac{X_{1: n}(1-\mathrm{p})^{-\frac{1}{\mathrm{c}}}}{\mathrm{nc}} ; \text { for } i=5 \\
\frac{1}{\mathrm{n}\left(\frac{\mathrm{X}_{1: \mathrm{n}}}{\mathrm{t}}\right)^{\mathrm{c}} ; \text { for } i=6} \\
-\frac{1}{\mathrm{n}}\left(\frac{\mathrm{X}_{1: \mathrm{n}}}{\mathrm{t}}\right)^{\mathrm{c}} ; \text { for } i=7
\end{array}\right.
$$

respectively.

Then by defining $M\left(\mathrm{X}_{1: \mathrm{n}}\right)$ given in (2.9) and substituting $\mathrm{T}\left(x_{1: n}\right)$ as appeared in (2.11) for (2.10), $\mathrm{f}(x, \theta)$ is characterized.

Example 3.3 In context of remark 2.2 characterization of Pareto distribution through $\mathrm{p}^{\text {th }}$ quantile $; \widetilde{Q}_{p}(\theta)$ is given.

$$
\tilde{Q}_{p}(\theta)=\theta(1-p)^{-\frac{1}{c}}
$$

Therefore

$$
g(\theta)=X(1-p)^{-\frac{1}{c}}
$$

and from (2.3)

$$
\phi(X)=g(X)-\left(\frac{X}{c}\right) \frac{d}{d X} g(X)=\left(\frac{c-1}{c}\right)(1-p)^{-\frac{1}{c} X}
$$

and (2.9) of remark 2.1

$$
M(\mathrm{X})=\frac{\frac{d}{d \mathrm{X}} g(\mathrm{X})}{\phi(\mathrm{X})-g(\mathrm{X})}=-\frac{\mathrm{c}}{X}
$$

with

$$
\frac{d}{d x_{1: n}}\left[\log \left(\frac{1}{c x^{c}}\right)\right]=M\left(\mathrm{X}_{1: \mathrm{n}}\right)
$$

then

$$
\mathrm{T}(X)=-\frac{1}{c x^{c}}
$$

and 


$$
\mathrm{f}(x, \theta)=-\frac{\frac{\mathrm{d}}{\mathrm{dx}} T(\mathrm{X})}{T(\theta)}=\frac{c \theta^{c}}{x^{c+1}}, \mathrm{a}<\theta<x<b .
$$

Example 3.4 Using remark 2.2 the pdf $\mathrm{f}(x, \theta)$ defined in (1.1) can be characterized through non constant functions of $\theta$ such as

$$
g_{i}(\theta)=\left\{\begin{array}{c}
\mu_{1}^{\prime}(\theta)=\frac{c}{\mathrm{c}-1} \theta ; \text { for } i=1 \\
\mu_{r}^{\prime}(\theta)=\frac{c}{\mathrm{c}-\mathrm{r}} \theta^{r} ; \text { for } i=2 \\
e^{\theta} ; \text { for } i=3 \\
e^{-\theta} ; \text { for } i=4 \\
Q_{p}(\theta)=\theta(1-p)^{-\frac{1}{c}} ; \text { for } i=5 \\
F(t)=1-\left(\frac{\theta}{t}\right)^{c} ; \text { for } i=6 \\
\bar{F}(t)=\left(\frac{\theta}{t}\right)^{c} ; \text { for } i=7
\end{array}\right.
$$

by using

$$
\left[\phi_{\mathrm{i}}\left(\mathrm{x}_{1: \mathrm{n}}\right)-g_{i}(\theta)\right]=\left\{\begin{array}{c}
-\frac{X}{c-1} ; \text { for } i=1 \\
-\frac{r X^{r}}{c-r} ; \text { for } i=2 \\
-\frac{X e^{X}}{c} ; \text { for } i=3 \\
\frac{X e^{-X}}{c} ; \text { for } i=4 \\
-\frac{X}{\mathrm{C}}(1-\mathrm{p})^{-\frac{1}{\mathrm{c}}} ; \text { for } i=5 \\
\left(\frac{X}{t}\right)^{c} ; \text { for } i=6 \\
-\left(\frac{X}{t}\right)^{c} ; \text { for } i=7
\end{array}\right.
$$

Then by defining $M\left(\mathrm{X}_{1: \mathrm{n}}\right)$ given in (2.9) and substituting $\mathrm{T}\left(x_{1: n}\right)$ as appeared in (2.11) for (2.10), $\mathrm{f}(x, \theta)$ is characterized. 


\section{Reference}

Ahsanullah, (1973). A characterization of the Pareto distribution. Canadian Journal of Statistics, Volume 1, Issue 1-2, pages $109-112,1973$

Ahsanullah (1974). Characterization of the Pareto distribution. communication in statistics, 3(10), 953-957, 1974.

Ali, M. A. and Khan, A. H. (1998). Characterization of Some Types of Distributions, Information and Management Sciences, Vol. 9 , No. 2, June, 1998.

Beg, M. L and Kirmani, S. N. U. A, (1974). On a characterization of exponential and related distributions, Austral. J. Statist., 16 (3), 1974, 163-166.

Dallas, A. C. (1976). Characterization Pareto and power distribution, Ann. Ins. Statist. Math, 28, (1976), Part A, pp. 491 - 497

Dimaki,C.and Evdokia Xekalaki. (1993). Characterizations of the Pareto distribution based on order statistics. Stability Problems for Stochastic Models, Lecture Notes in Mathematics Volume 1546, pp 1-16.

Henrick John Malik, (1970). A characterization of the Pareto distribution. Scandinavian Actuarial Journal, Volume 1970, Issue 3-4, 1970.

Krishnaji, N, (1970). Characterization of the Pareto Distribution Through a Model of Underreported Incomes. Vol. 38, No. 2, Mar., 251-255, 1970.

Nagesh S. R., Michael J. H. and Marcello P. (1974). A Characterization of the Pareto Distribution. Institute of Mathematical Statistics Vol. 2, No.3, Page 599 of 599-601, 1974.

Shah, S. M. and Kabe, D. G, (1981). Characterizations of Exponential, Pareto, Power Function, BURR and Logistic Distributions by Order Statistics. Biometrical Journal, volume 23, Issue 2, pages141-146.

Srivastava, M. S. (1965). Characterizations of Pareto's Distributions and $(k+1) x^{k} / g^{k+1}$. Ann. Math. Statist.,36, 361-362.

Tavangar, M. and Asadi, M. (2019). some new characterization results on exponential and related distributions, Bulletin of the Iranian Mathematical Society Vol. 36 No. 1 (2010), pp 257-272. 